Canadian University Music Review

Revue de musique des universités canadiennes

\title{
Petite histoire de mon théâtre musical
}

\section{Michel Puig}

Numéro 3, 1982

URI : https://id.erudit.org/iderudit/1013832ar

DOI : https://doi.org/10.7202/1013832ar

Aller au sommaire du numéro

Éditeur(s)

Canadian University Music Society / Société de musique des universités

canadiennes

ISSN

0710-0353 (imprimé)

2291-2436 (numérique)

Découvrir la revue

Citer cet article

Puig, M. (1982). Petite histoire de mon théâtre musical. Canadian University Music Review / Revue de musique des universités canadiennes, (3), 158-169.

https://doi.org/10.7202/1013832ar

All Rights Reserved (C Canadian University Music Society / Société de musique des universités canadiennes, 1982
Ce document est protégé par la loi sur le droit d'auteur. L'utilisation des services d’Érudit (y compris la reproduction) est assujettie à sa politique d'utilisation que vous pouvez consulter en ligne.

https://apropos.erudit.org/fr/usagers/politique-dutilisation/ 


\title{
PETITE HISTOIRE DE MON THÉÂTRE MUSICAL*
}

\author{
Michel Puig
}

Le théâtre musical, locution passe-partout appartenant à la terminologie actuelle des compositeurs, musicologues et critiques, semble être encore à la recherche de son identité. Objet en devenir, en mutation constante, il appelle l'analyse mais défie la synthèse. Objet pluriel dans sa formulation même, il se présente au premier regard semblable à un corps céleste formé de deux hémisphères jumeaux : non point dichotomie théâtre/musique mais, bien au contraire, fusion de ces deux éléments pour générer deux combinaisons distinctes et spécifiques.

L'une de ces combinaisons, face la plus visible du théâtre musical, répond au souci de nombreux compositeurs qui trouvent dans la théâtralisation de leur musique des solutions à des problèmes compositionnels ; elle privilégie une forme scénique nouvelle dont l'axe premier, le moteur, est la musique. Il serait mal venu de ma part de me substituer à ces compositeurs pour décrire et expliciter leur démarche ; telle n'a jamais été mon approche du Théâtre musical, aussi, n'en dirai-je rien. L'autre combinaison représente la face cachée de notre corps céleste, celle dont le geste théâtral repensé dans des structures musicales est la raison d'être et qui ne se laisse appréhender que par une longue pratique expérimentale. C'est de celle-ci que je désire vous entretenir. Ainsi, analysant les étapes d'une recherche de plusieurs années, j'espère vous amener à découvrir comment la musique a ouvert au théâtre des perspectives nouvelles.

Pour moi, tout commence en 1960. Paris est à cette époque le haut lieu de la recherche et de la réalisation dramatiques. C'est le

*Ce texte constitue la version, revue et mise en forme par Marthe Forget, d'une conférence donnée par Michel Puig le 8 octobre 1981 à la Faculté de Musique de l'Université de Montréal, à l'occasion d'une visite d'un mois au cours delaquelle il a réalisé, avec les étudiants de l'atelier de jeu scénique et de l'atelier de musique contemporaine, "Le rêve du papillon ", conte chinois du XIIIe siècle.

Revue de musique des universités canadiennes, $\mathrm{N}^{\circ} 3,1982$. 
théâtre à thèse de Sartre, de Camus, de Gabriel Marcel ; c'est aussi le théâtre néo-classique de Montherlant et celui des romanciers comme Mauriac et Bernanos. Si le théâtre d'Audiberti et de Guelderode appartient encore au cénacle de l'avant garde, le théâtre de l'absurde, lui, s'impose à un plus large public: Becket, Ionesco, Adamovet Tardieu sont joués au théâtre de Babylone par Jean-Marie Serreau et Roger Blin. Au zénith de cette intense activité théâtrale, le T.N.P. de Jean Vilar brille de tous ses feux. Cet te même année, je participe à une soirée non-stop au musée des Arts Décoratifs où, durant plusieurs jours, musique, danse, cinéma et théâtre se succéderont en séquences assez brèves. Le temps fort de cet événement reste pour moi la création d'une pièce de Becket : Comédie, mise en scène par J.M. Serreau. J'ai, pour la première fois, la révélation d'un véritable théâtre musical.

Le texte de cette pièce est provocant, dit d'une manière totalement nouvelle, dans une mise en scène qui, radicale, va jusqu'au bout de ses intentions. Trois têtes émergent de grosses jarres où sont enfermés les personnages interprétés par Delphine Seyrig, Emmanuelle Riva et Michaël Lonsdale. Un projecteur unique, passant d'un visage à l'autre, découpe le dialogue : une voix sort de l'ombre et parle ... abandonnée par la lumière, elle se tait. Ainsi, toute la puissance expressive du comédien se trouve concentrée dans le visage et la voix ; l'absence de gestuelle ainsi qu'une diction entièrement fabriquée, recomposée, favorisent cette concentration. La diction, anti-naturelle, est à la voix ce que les jarres sont au corps : elle lui retire toute possibilité d'expression. Le texte, chanté dans un tempo très accéléré, inusité au théâtre, doit être répété car l'absence de tout silence et la précipitation du discours rendent incertaine, à la première écoute, la compréhension du texte. Or, de quoi s'agit-il ici, sinon de faire passer un texte? Un beau texte porteur de sons, de rythmes, d'images, un texte où les mots font mouche, deviennent les pierres à sucer de Molloy! Le théâtre de Becket se passe de déplacements, de gestes, de costumes, de décor, de lumière, de musique. Il peut se passer de tout, sauf de mots et de comédiens pour les dire. Plus tard, il se passera parfois de mots, mais jamais de comédien tant il est vrai que le théâtre, c'est avant tout un comédien devant un public.

Mais, à ce texte, quels chemins ces comédiens peuvent-ils frayer en eux mêmes, pour qu'il y éveille un écho authentique et revienne, ainsi vécu, aux spectateurs? Aucun, répondent auteur et metteur en scène. A ce théâtre différent, donnons une diction 
autre; à des personnages qui n'en sont pas, donnons une parole qui n'en soit pas une, antinomique, et rendons-la aussi artificielle que le texte lui-même. Définir pour chaque syllabe, pour chaque mot, un timbre, un rythme, une hauteur, une intonation, voilà le travail hors de l'ordinaire auquel se sont appliqués comédiens, metteur en scène et auteur. Sans aucun doute, c'est de chant qu'il s'agissait ; mais pour qu'il soit tout à fait musique, il manquait à ce chant une dimension importante : une véritable structure musicale. C'est pourquoi sans doute cette tentative de Serreau est restée sans lendemain. C'est pourquoi je vais me sentir, dès lors, concerné par la structure musicale d'un texte dramatique quel qu'il soit.

Ceci m'amène à vous parler de la première étape de ma recherche qui fut consacrée à la problématique du chant. Je donne au mot chant une acception large : on chante dès l'instant où l'on s'aventure hors du chemin étroit du langage parlé. Plus précisément : on chante lorsqu'on assume consciemment les hauteurs, la durée et la rythmique du son de sa voix ; par le fait même sont exclus le cri et la parole. Chanter et parler sont comme vers et prose ... il suffit de ne plus être en état de chant pour que le parler revienne.

En 1967, Colette Magny, chanteuse bien connue en France pour sa voix ample et originale, sa personnalité et son engagement politique, me manifesta sa lassitude d'interpréter des chansons traditionnelles. Mais comment pouvait-elle renouveler son répertoire? Devait elle s'appliquer, comme bien des chanteurs, à devenir compositeur ou, pouvions-nous, par une recherche commune, trouver une voie nouvelle? Je lui exposai alors ma façon de concevoir le chant qui reçut son approbation et je lui proposai d'inventer un chant avec elle sur des textes que nous choisirions ensemble. Le premier fut un poème de Max Jacob: Les Malachites. C'est un exemple parfait de ce que l'auteur lui-même appelle « le cubisme poétique "; une rythmique très savante le structure en petites séquences courtes, discontinues. L'imagination musicale ne peut que suivre. Nous pouvions donc nous laisser porter par les images.

Quel fut mon rôle? D'abord, assez négatif car je refusais le plus souvent ce que Colette Magny proposait. Je lui demandais de dire le poème comme elle le sentait ; mais sa voix restait celle de ses tours de chant, parfaitement adaptée aux chansons qu'elle avait déjà chantées et dont elle ne voulait plus : les formules mélodiques restaient banales, issues d'une harmonie stéréoty- 
pée. En effet, toute improvisation tend à faire réémerger le déjà entendu qu'il convient précisémet d'évacuer. Parce qu'elles impliquent une harmonie, nous renonçâmes aux notes proprement dites et, à la manière des musiciens sériels, nous mîmes en pratique, suivant ma méthode, la décomposition du son en ses différents paramètres. Je conseillai à Colette Magny de se servir des caractéristiques suivantes : timbre, attaque, dynamique et durée. Je l'aidai à prendre à son compte les registres, la respiration contrariée, les bruits produits par le corps : il fallait que cela devînt sa propre invention. Ainsi, il lui serait impossible de chanter faux comme on dit d'un comédien qu'il parle faux. Le son juste devenait celui auquel sa nature profonde, en s'exprimant, donnait sa forme. Nous en arrivâmes très rapidement au travail du mot à mot. Je poursuivais ainsi la recherche de Jean-Marie Serreau, en la soumettant en outre à mon expérience de compositeur. Mon rôle consistait donc à maintenir Colet te Magny dans cet éfat de vérité. Je l'aidai également à préciser et à noter l'acquis, à le sélectionner et à le coordonner afin que, de cette investigation intérieure, naquît une chanson susceptible de figurer à son répertoire. Ayant réussi, nous poursuivîmes l'expérience avec un poème de Lewis Carroll, le célèbre Jaberwocky chanté en anglais, profitant de la liberté sémantique de ce poème du "non-sens " pour nous laisser aller aux mots, à leur saveur, à leur jeu ... (aventure verbale qui trouva d'ailleurs un écho l'année suivante à l'occasion d'un travail réalisé avec l'écrivain et musicologue Alejo Carpentier pour qui la mise en musique d'un texte impliquait que celui-ci répondit à certaines pertinences : expression forte, syntaxe brisée, enchaînements rares ou inexistants, des verbes, des substantifs, peu d'adjectifs, aucun mot adventice, du rythme).

Nous nous trouvions néanmoins très loin de l'improvisation car nous avions constaté que la mise en oeuvre d'un chant de ce type ne progresse qu'au prix d'une extrême tension et à travers de longs tâtonnements; mais, une fois définis et considérés comme justes, le mot et/ou la phrase vont résister à toute tentative de modification. Si certains éléments peuvent varier, disparaître même au cours des répétitions, d'autres en revanche, semblent inamovibles : ils deviennent l'essence même de la chanson qui, bien des années plus tard, sans avoir été notée restera en mémoire. De nombreux comédiens ou étudiants depuis ont chanté ces chansons et, à ma grande surprise, à travers des interprétations fort différentes, l'objet vocal persiste d'un chanteur à l'autre. J'en suis donc bel et bien le compositeur. Pourtant, au cours de notre 
cheminement expérimental, l'acte de composer a changé de nature, modifiant le rapport de l'interprète au compositeur : celui-ci, dans une attitude d'écoute passionnée, a pris en charge l'invention musicale de celui-là. Mais en dépit de cette altérité, le produit fini demeure bien l'oeuvre du compositeur. C'est ainsi qu'il faut comprendre la démarche d'un Duke Ellington qui composa la musique la plus personnelle qui soit grâce à des musiciens qui ne furent pourtant jamais plus eux-mêmes que dans son orchestre. Dans cette échange, chacun trouve son compte et participe à la création . L'interprète cesse d'être un subalterne au service du pouvoir que détient le compositeur.

Je n'ai jamais travaillé autrement depuis, avec des comédiens, des chanteurs ou des instrumentistes, des éclairagistes ou des décorateurs. Cependant, j'ai eu besoin d'un long temps d'apprentissage pour prendre conscience que ce rapport interprète/auteur constituait l'épicentre de l'art théâtral et que ma nouvelle manière de composer participait précisément du théâtre musical.

D'autres, heureusement, l'ont compris pour moi ... En 1969, sur les conseils de Jean-Pierre Drouet avec qui je travaillais depuis longtemps, Jean-Marie Serreau m'invita à rejoindre sa compagnie théâtrale. Il montait alors une adaptation par Aimé Césaire de La tempête de Shakespeare. Ma tâche consistait à faire chanter, à ma façon, quelques textes qui jalonnaient la pièce. Parmi le groupe de musiciens engagés, outre Jean-Pierre Drouet et Michel Portal, se trouvait le très remarquable jazzman martiniquais Eddy Lowiss et le batter haïtien Cheriza. Le metteur en scène semblait dépassé par l'extraordinaire outil à produire de la musique que nous constituions. Il ne savait que nous demander ; nos propositions allaient toujours au-delà de ce qu'il souhaitait et la musique ne parvenait pas à s'intégerer à la réalité du spectacle.

Mais il convient ici de parler de Michaël Lonsdale ; vous ne pouvez imaginer en effet le besoin d'invention qui l'habite. Dans La tempête, il jouait le rôle de Prospero et je dois avouer qu'il tenait le spectacle à bout de bras. Lors d'une répétition, Lonsdale me confia qu'il ne supportait plus d'interpréter le monoloque au cours duquel Prospero faisait, très longuement, le récit de sa vie ; et de fait, cette narration était chaque soir un des temps morts de la pièce. Ne voulant plus continuer ainsi, il me demanda de lui donner les moyens de chanter ce texte, à ma manière bien entendu ; je lui proposai de l'accompagner en grattant avec des clous les cordes du piano.

Nous avions préparé notre numéro dans le plus grand secret, 
aussi, lorsque nous nous exécutâmes, la stupéfaction fut-elle totale sur le plateau et même dans la salle. Mais Lonsdale a un sens très vif de la trouvaille vocale - qu'on se souvienne d'India Song - et le public accueillit avec enthousiasme cette innovation.

Nous ne pouvions pas en attendre autant de Césaire qui voyait une remise en question de son texte dans le détournement que nous lui faisions subir. Césaire tenait à ce texte plus qu'à son drame; nous, nous tenions à notre musique. Ainsi se révélait une difficulté majeure, sournoise parce que parée d'une fausse évidence : le théâtre considéré comme la manifestation d'un phénomène prioritairement littéraire. En effet, la notion selon laquelle le théâtre depuis ses plus antiques origines appartient au domaine des écrivains de pièces est si largement répandue que des metteurs en scène aussi remarquables que J.M. Serreau et Marcel Maréchal on pu affirmer publiquement que sans texte il n'y avait pas de théâtre.

Il me paraissait cependant évident qu'un texte organisé, fermé sur lui-même, d'une part et, d'autre part, une musique très composée ne pouvaient coexister dans une même structure sans que l'un des deux ne prît le pas sur l'autre : la prégnance de leurs formes respectives se révélait trop contraignante. C'est donc à l'invention dramatique que nous demanderons d'être à l'origine et au centre de la création, faisant appel - ou non - à des paramètres musicaux ou à ceux du discours littéraire. Et voici que se révèle insensiblement la face cachée de notre théâtre musical.

Pendant trois ans, je n'ai pas cessé de poursuivre la recherche commencée avec Colette Magny ; la troupe de Serreau se réunissait une fois par semaine pour travailler avec moi ; en 1970, ce fut celle de Catherine Dasté et un peu plus tard, la compagnie de Claude Régy. Je donnais également des cours à l'école dramatique de Tania.Balachova auxquels s'ajoutèrent ceux des Université de Paris VIII-Vincennes et Paris X-Nanterre où j'enseigne encore aujourd'hui. Nombreux sont les textes que nous avons soumis à nos pratiques! Je pressentais que nous trouverions ainsi la réponse à nos questionnements.

Après le demi-échec de La tempête s'amorça une longue collaboration entre Catherine Dasté et moi-même ; j'aime son théâtre, sa poésie, ses réalisations, son interprétation mythique de la scène; j'aime aussi ses textes (quand il y en a ... car ils sont rares!). D'une répétition à l'autre, je découvrais que sa manière de mettre en scène ses spectacles, impalpables et forts, s'apparentait beaucoup à la méthode que j'avais mise au point pour faire 
chanter les comédiens et jouer les musiciens : même rapport de collaboration entre elle et ses interprètes, même réticence à l'égard de l'improvisation, mais aussi, même refus de subordination à la chose écrite. Personne, cependant, ne lui conteste le droit de signer ses spectacles tant il est vrai qu'ils ne ressemblent à aucun autre. De Catherine Dasté, j'ai appris que l'acte essentiel du théâtre que nous recherchions l'un et l'autre, l'acte créateur, premier, était celui du dramaturge qui assume dans sa totalité le déroulement du spectacle. La dramaturgie dispose du texte et de la musique, elle les commande et les organise ; elle détermine décors, costumes et éclairages et elle donne vie au jeu des acteurs.

Ainsi, devenu auteur de pièces mais non écrivain, j'ai réalisé une vingtaine de spectacles dont je n'ai écrit ni les phrases ni les mots. J'ai adapté de nombreux livres et parfois imaginé moi-même des arguments dont j'ai confié la rédaction à quelques auteurs. Mais j'ai aussi, avec certains d'entre eux, tenté cette création commune qui s'avère de plus en plus nécessaire à la réalisation d'un théâtre musical. A cet égard, mon travail avec Michel Leiris puis avec Robert Pinget me paraît significatif. En 1974, je proposai à Leiris de réaliser un spectacle d'après son livre Nuits sans Nuit. L'oeuvre et la démarche de cet écrivain trouvent chez moi un écho profond. Je choisis donc sept des récits de rêves qui constituent Nuits sans Nuit et organisai leur déroulement à la façon d'une longue narration qui révélerait petit à petit l'univers du poète. Lonsdale assurait la mise en scène. Au cours du travail, il nous parut impossible de faire chanter ces rêves tels que Leiris les avait écrits. Aussi, avons-nous choisi les mots, les bribes de phrases qui répondaient davantage à notre projet. Une fois le travail commencé, nous invitâmes Leiris à donner son avis, très inquiets de sa réaction... Il avait, dit-il, noté ses rêves dans le seul but de les arracher à l'oubli ; poète, il les avait métamorphosés en poèmes. Aussi ne fut-il pas choqué de la mutilation que nous infligions à son texte : le spectacle, par ses ruptures textuelles, sonores et visuelles restituait, pour lui, le déroulement onirique dans son instantanéité.

Plus tard, je réalisai deux pièces différentes et un film tirés d'une oeuvre de Robert Pinget: Graal Flibuste. La première pièce fut créée en Avignon après un stage de travail intensif d'une quinzaine de jours durant lesquels Pinget accepta d'écrire un texte dramatique dans le pas à pas de l'évolution du spectacle, s'adaptant non seulement aux exigences scéniques, mais tenant compte aussi de la nature profonde de chaque comédien qui y 
participait. Faut-il préciser que dans les deux spectacles le texte était entièrement chanté? En tant que compositeur, je me heurtai donc aux difficultés inhérentes à toute structure musicale mais, responsable désormais de l'ensemble des éléments dramatiques, je pus enfin introduire comme paramètre sonore indispensable à la dramaturgie une musique qui s'inscrivait dans le droit fil de mes recherches de compositeur.

Cette expérience se concrétisa encore davantage en 1972 avec une adaptation de La Chasse au Snarck de Lewis Carroll ; Catherine Dasté en avait fait la mise en scène pour le festival d'Avignon et nous la jouâmes ensuite à Paris. Un texte anglais, comportant des indications de hauteurs précises, se mêlait à un texte français dont le déroulement musical restait libre. Afin de conserver la richesse de timbres des expressions et la spontaneité du jeu, je confiai le texte anglais à de jeunes comédiennes dont la voix n'était ni travaillée, ni posée. Durant plusieurs mois elles s'employèrent à mémoriser le texte auquel j'avais donné une véritable texture musicale. L'écriture vocale jouait sur un registre extrêmement étendu : ainsi nous évitions le danger d'une tessiture homogène de la voix qui fait souvent écran au caractère dramatique du personnage. Les comédiennes choisissaient expressions et timbres divers, mais toujours dans le respect des hauteurs que j'avais indiquées. La participation d'ungroupe de musiciens assurait la présence d'une structure harmonique.

Je n'étonnerai personne en disant que les notes des comédiennes étaient approximatives : mais, au fil des représentations (une cinquantaine environ), la justesse d'intonation finit par s'imposer. L'écriture musicale du texte français fut confiée à l'inspiration d'un deuxième groupe de comédiens ; facteur très important de renouvellement pour les interprètes, cette invention permanente entretenait aux cours des représentations un caractère d'immédiat.

Peu après la mort de J.M. Serreau qui avait accueilli dans son théâtre notre Chasse au Snarck, Michaël Lonsdale et moi décidâmes de travailler en collaboration : lui, comme metteur en scène et acteur, moi, comme auteur et musicien. En trois ans nous avons créé cinq spectacles. Dans la chaîne complexe de chacune de ces dramaturgies, je continuai à glisser les fils fragiles de la tramedes divers paramètres musicaux. Dans la mesure où je n'entravais pas le libre jaillissement expressif du texte, je lui appliquais ma propre écriture vocale. Je poursuivais la même démarche sur la plan rythmique : ainsi plusieurs tentatives furent-elles faites 
d'associer dans un même segment rythmique le vocal et le gestuel. D'abord restée sans succès, l'expérience réussit pleinement dans Nuits sans Nuit. Sur la scène, éclairés par la seule lueur des bougies, deux comédiens sont agenouillés. A gauche, un comédien met en mouvement un métronome. Il prononce chaque syllabe du texte en respectant les silences et les hauteurs que j'ai moi-même fixés sur le manuscrit. Simultanément, en suivant la battue métronomique (un temps sur deux), les yeux du comédien vont de gauche à droite puis de droite à gauche. La comédienne placée à droite tient dans la main un petit métronome qu'elle peut accélérer d'une simple pression. A son tour elle dit le texte, bougeant également les yeux mais cette fois à chaque battue du métronome. Elle accélère peu à peu le tempo de l'énonciation du texte, guidée par le métronome que le public n'entend pas alors que le premier demeure constamment audible. Les textes se chevauchent et les yeux, toujours en mesure, sont animés d'un mouvement de plus en plus rapide qui aboutit à un tournoiement. Ainsi, grâce a ce jeu d'alternance rythmique entre silence et énoncé syllabique, un climat d'angoisse s'installe et grandit au fur et à mesure que se déroule le récit du rêve : une escalade nocturne parmi des nids de vautours. De fait, c'est une véritable pensée contrapuntique - cette composante fondamentale de la musique - qui organise ces quatre voix (deux chants, deux regards mobiles) et qui devient élément dynamique de l'expressivité théâtrale.

Dans le spectacle suivant, Fragments pour « Che » Guevara, le contrepoint fut greffé d'une façon fort différente sur le texte de Pierre Bourgeade: ici, Michaël Lonsdale était comédien et metteur en scène, j'étais compositeur et comédien. Le violoniste Régis Pasquier s'était joint à nous. Je lui avais donné un canevas harmonique, mélange d'itinéraire et d'incitation à l'invention, basé sur une conception assez particulière de l'harmonie à laquelle je tiens assez pour la placer désormais au centre de mon écriture musicale. Lonsdale et Pasquier tiraient tous deux de leur instrument les accents les plus émouvants comme les plus comiques, repoussant l'étendue des registres et les contrastes dynamiques dans leurs limites les plus extrêmes.

A travers ces multiples expériences, j'étais devenu à la fois compositeur, concepteur et comédien. Il ne manquait à ma panoplie d'auteur de théâtre musical que la pratique de la mise en scène et celle de la direction d'acteurs. Auprès de J.-M. Serreau, puis de Catherine Dasté et enfin de Claude Régy, j'avais accumulé suffi- 
samment d'expérience pour me permettre d'aborder sans trop de risque ce complexe et difficile métier. Je commençai en 1975 au cours d'un stage que j'avais organisé en Avignon.

Que voulais-je obtenir de mes comédiens? Qu'ils mettent toute la force de leur concentration intérieure au service de la dramaturgie. Durant de très longues répétitions, j’avais vu Claude Régy, impitoyable, obliger les comédiens à se lancer à la poursuite d'eux-mêmes dans une chasse sans merci : introspection difficile qui se traduisait parfois par des crises d'une violence inouïe chez les interprètes. Emergeait alors à la surface de ce maelstrom intérieur, comme suspendue dans le vide, une étrange diction qui possédait la force de l'évidence.

Dans notre travail commun, Lonsdale essaiera d'obtenir, par d'autres moyens, cette même concentration. Fort de ces expériences théâtrales ainsi que de son métier de peintre, Lonsdale dirigera déplacement et gestuelle de l'acteur avec une minutie qui peut être considérée comme totalitaire tant il aura tendance à écarter toute initiative autre que la sienne. Les comédiens seront ainsi posés sur la mise en scène comme les lignes et les couleurs le sont sur une toile.

Lorsqu'est venu mon tour de diriger des comédiens, j'ai donc essayé de poursuivre dans le même sens ; mais, profitant de l'expérience que j'avais de l'acteur-proférateur de son, j'y ajoutai une gestuelle entièrement élaborée comme une musique. Je demandai moi aussi aux comédiens un contrôle absolu de leur corps, comme on exige d'un instrumentiste le contrôle de son instrument. Mesurer la durée des gestes, maîtriser leur espace et leur intensité : tout instrumentiste en fait l'apprentissage. Pas le comédien. C'est en lui imposant cette contrainte que naîtra peu à peu un comédien autre. En effet, le problème du jeu de l'acteur est inversé par rapport à celui du musicien ; celui-ci est formé pour jouer une partition à l'élaboration de laquelle il reste étranger : on lui remet cette partition et il l'exécute. Afin de libérer sa créativité, il faudra l'obliger à se passer de ce support. L'acteur, lui, est conditionné par des stéréotypes qui relèvent souvent de modes d'improvisations ou du gestuel traditionnel des emplois. Il construit souvent son jeu sur ces fausses prémisses ; souvent aussi selon l'inspiration du moment.

Certes, introduire les paramètres musicaux pour saisir ce jeu, comme l'instantané photographique fixe le mouvement, peut paraître une véritable contrainte. C'est ainsi néanmoins que, privée de ses issues habituelles, la vie afflue à nouveau dans le jeu des acteurs, activant des zones inattendues de la créativité. 
L'approfondissement de ces techniques fit l'objet de ma recherche en théâtre musical dont la dernière étape fut la réalisation d'un spectacle créé en 1979 aux semaines musicales d'Orléans. Il s'agit d'une longue réflexion sur la préoccupation fondamentale du peintre Claude Monet. Pourquoi, chez lui, cette poursuite forcenée de l'apparence? Qu'y cherchait-il? Pendant près de soixante-dix ans sa peinture ignore le reste du monde. Quelle fut la force de cette fascination pour que la Commune, les mouvements ouvriers et nationalistes, la guerre de 14-18, la révolution d'octobre, ne trouvent aucune place dans l'oeuvre de Monet? Cette interrogation a donné naissance à un théâtre musical en quatre tableaux : Monet ou la passion de la réalité. Une analyse de ce spectacle ne me paraît pas inutile : elle me permettra de compléter et de résumer les propos qui ont précédé.

Premier tableau : Monet, confronté à la peinture réaliste de son époque. Camille, sa femme, pose pour lui, contrefaisant des attitudes célèbres : elle est tour à tour le modèle de l'Atelier de Courbet, l'Olympia de Manet, la Femme au tub et la Repasseuse de Degas. Le peintre s'en détourne; il fixe au fond de la scène un écran où s'animent des images d'eau. « Folie de vouloir tout capter ... il faut peindre la joie », dit Camille. Mais devant la grande femme noire, image tragique de la Commune, elle sort en pleurant. La réponse de Monet apparaît sur l'écran : Impression du soleil levant (1875).

Deuxième tableau : Monet est placé devant une autre facette de la réalité: un peintre doit-ilêtre le témoin de son temps? Un très rapide roman-photo défile en images désarticulées qui sont projetées sur des voiles de bateaux. Nous sommes en 1914 ; c'est le départ d'un bel officier pour le front. Monet en retient la dernière image : Le retour de la gare. Est-ce le matin ou le soir? Le temps est-il clair ou brumeux? Il repeint plusieurs fois le même sujet. Il invente ainsi les séries ... Les Cat hédrales de Rouen apparaissent sur l'écran.

Troisième tableau : Camille suggère à Monet de peindre son portrait. Monet reste immobile regardant les fleurs. Tout s'assombrit et ralentit. "A force de peindre la vie, on rencontre la mort ". La scène est d'un bleu très léger, presque obscure. Camille la traverse lentement et se couche. En effet, le vrai portrait de sa femme est celui que Monet a peint devant son lit de mort. La fin de la scène montre ce tableau terrible. Monet le regarde et peu à peu Camille, comme Ophélie, flotte à la surface de l'eau.

Quatrième tableau : le film jusqu'alors en noir et blanc 
devient d'un bleu intense. Face à l'image de la mer, le peintre reste seul, enfermé dans sa méditation, à la poursuite du reflet des choses. Peu à peu, la longue série des Nymphéas apparaît sur l'écran.

De cet argument j'ai fait une sorte de symphonie en quatre mouvements (sous-titre du spectacle) : allegro moderato, scherzo, adagio, finale. Au début de chaque mouvement, le résumé de l'action, inscrit sur des diapositives, apparaît sur l'écran ; la lumière revient sur le plateau, les comédiens toussent, les musiciens s'accordent. Cette petite intrusion dans la sociologie des concerts, comme cette mise en forme symphonique du spectacle, ne servent qu'à informer le spectateur sur les intentions de l'auteur qui tient à dire : «ce spectacle est aussi musique ». Bien entendu, la réalité musicale est ailleurs ...

Pour la première fois, je pense avoir réuni en un tout homogène les composantes visuelles, sonores et gestuelles d'un spectacle en maintenant chacune d'elle à son point le plus élevé d'expressivité. Comédiens, texte, musique, film, diapos, éclairages sont, à part entière, les sept voix d'un contrepoint qui, du début à la fin, tisse la matière même du spectacle.

Seul un compositeur, après une longe pratique de son métier, peut mener à bien une polyphonie théâtrale; mais combiner des éléments aussi divers et autonomes que la mise en scène, le jeu des acteurs, la musique, la projection des couleurs, des images et des éclairages, exige une profonde connaissance de leur fonctionnement intrinsèque. C'est à ce prix que j'ai obtenu mon véritable outil de travail: la dramaturgie, partition multidimensionnelle du théâtre musical. 